\title{
Profile of microRNAs in the plasma of Parkinson's disease patients and healthy controls
}

\author{
Lucía F. Cardo $\cdot$ Eliecer Coto $\cdot$ Lorena de Mena \\ Renée Ribacoba · Germán Moris · Manuel Menéndez · \\ Victoria Alvarez
}

Received: 13 February 2013/Revised: 18 March 2013/Accepted: 19 March 2013/Published online: 30 March 2013

(c) Springer-Verlag Berlin Heidelberg 2013

\section{Dear Sirs,}

The pathological hallmark of Parkinson's disease (PD) is the loss of dopaminergic neurons and the presence of Lewy bodies and Lewy neurites in the substantia nigra pars compacta, corpus striatum and brain cortex [1]. PD is a complex disease caused by the interaction of genetic/ inherited and environmental/acquired risk factors [2]. MicroRNAs (miRNAs) are small RNAs that control gene expression by binding to the $3^{\prime}$ UTRs of mRNAs [3]. Postmortem analysis of brain tissues and in vitro studies have identified several miRNAs implicated in PD. MiR-133b was shown to be downregulated in PD brains and to promote the survival of dopaminergic neurons [4]. MiR-433 was related with PD through targeting the FGF20, which in turn regulates the expression of $\alpha$-synuclein [5]. MiR-7 targets $\alpha$-synuclein and could regulate oxidative stress and cell death, while miR-184 and let-7 regulate dopaminergic neurons survival and activity [6, 7]. Recently, a miRNA

Electronic supplementary material The online version of this article (doi:10.1007/s00415-013-6900-8) contains supplementary material, which is available to authorized users.

L. F. Cardo - E. Coto $\cdot$ L. de Mena · V. Alvarez

Genética Molecular-Laboratorio de Medicina,

Hospital Universitario Central de Asturias, Oviedo, Spain

E. Coto

Department of Medicine, University of Oviedo, Oviedo, Spain

R. Ribacoba · G. Moris · M. Menéndez

Neurología, Hospitales Universitario Central Asturias

and Álvarez Buylla-Mieres, Asturias, Spain

V. Alvarez $(\bowtie)$

Genética Molecular, Hospital Central Asturias-Maternidad,

33006 Oviedo, Spain

e-mail: victoria.alvarez@sespa.princast.es profiling of PD brains identified early downregulation of $\mathrm{miR}-34 \mathrm{~b} / \mathrm{c}$, modulating mitochondrial function in areas with pathological affectation [8].

Plasma (circulating) miRNAs have been proposed as biomarkers for several diseases and aging $[9,10]$. Our aim was to characterize the plasma miRNA profile in PD patients and healthy controls, to determine its usefulness as a biomarker for PD. The study was approved by the Ethical Committee of Hospital Universitario Central de Asturias (HUCA) in accordance with the ethical standards of the Declaration of Helsinki and all the participants signed an informed consent. The study cohort consisted of sex- and age-matched healthy controls $(n=25$; mean age 67.6; $52 \%$ males $)$ and patients $(n=31$; mean age $63.9 ; 55 \%$ males) who fulfilled the PD-clinical diagnosis criteria [11]. None of the patients were receiving drugs for PD-treatment or had a diagnosis of cardiovascular or tumor disease.

Full details of the experimental procedure are available as supplementary material. Briefly, blood was collected in tubes with EDTA, centrifuged and plasma was aliquoted $(350 \mu \mathrm{l})$. A total of $2 \mathrm{pg}$ of a synthetic Arabidopsis thaliana miRNA (Ath-miR-159a; $5 \mu \mathrm{l}$ of a $0.4 \mathrm{pg} / \mu \mathrm{l}$ dilution) was immediately added and each aliquot stored at $-80{ }^{\circ} \mathrm{C}$ until use. Ath-miR-159a was used as control of the extraction process (supplementary material). Total plasma RNA was extracted (TRIzol ${ }^{\circledR}$ LS Reagent, Ambion) and resuspended in $25 \mu \mathrm{l}$ of RNAse-free water. Five $\mu \mathrm{l}$ of each sample were retrotranscribed (RT) with the Megaplex RT primers Human pool A and TaqMan microRNA Reverse transcription kit (Applied Biosystems). Three $\mu$ l of the RT product were pre-amplified with the Megaplex Preamp primers Human pool A and TaqMan Universal Master Mix no AmpErase UNG (Applied Biosystems). All the preamplifications were assayed with a custom Ath-miR-159a Taqman assay in an ABI 7500 Real-Time PCR (Applied 
Biosystems) and only samples with CTs in the range of 20-26 cycles were further processed. Thus far, no reliable endogenous control miRNA has been established to normalize plasma miRNA content, although several have been proposed. We assayed two normalization candidates (miR-191 and miR-223) and found that miR-191 was constitutively amplified in our samples showing the lowest inter-individual variability in patients and controls. All the expression values were thus normalized against miR-191.

In a first step, we searched for plasma PD-candidate miRNAs by analysing 384 miRNAs from three pools of PD

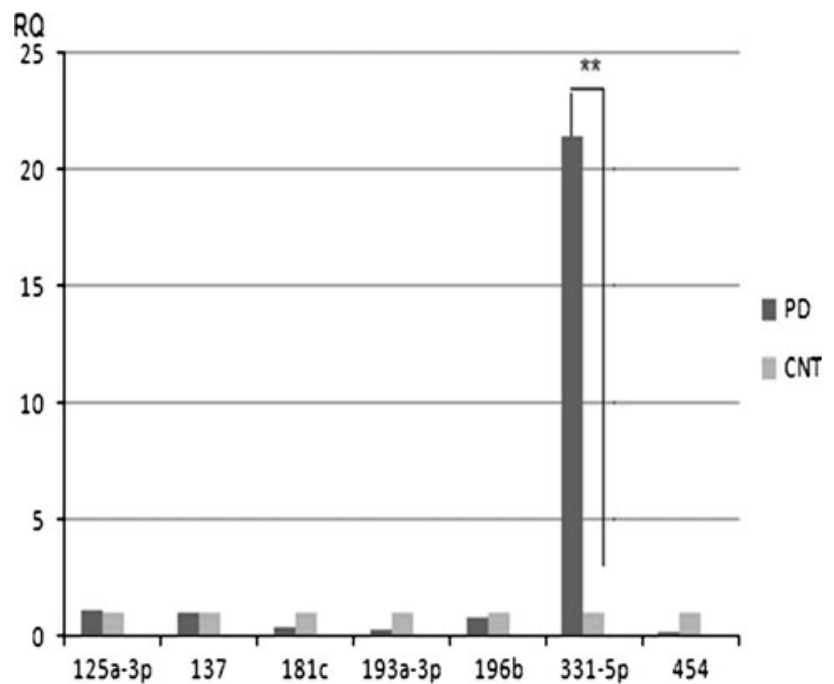

Fig. 1 Mean RQ values for the seven candidate miRNAs in PD $(n=31)$, and controls (CNT; $n=25)$. Plasma levels were normalized against the miR-191. Only miR-331-5p showed statistically significant difference between the two groups $(p=0.001)$. $\mathrm{RQ}=2^{-\Delta \Delta \mathrm{Ct}}, \quad \Delta \mathrm{Ct}=(\mathrm{Ct}$ miRNA $-\mathrm{Ct}$ miR-191 $), \Delta \Delta \mathrm{Ct}=(\Delta \mathrm{Ct}$ miRNA $-\Delta \mathrm{Ct}$ controls pool) patients and three pools of controls. Each pool contained pre-amplifications of four individuals, and were assayed with TaqMan low density miRNA (TLDA) cards on an ABI 7990 HT Fast Real-Time PCR equipment (Applied Biosystems). The amplification of each miRNA was normalized against miR-191. The following miRNAs were significantly overrepresented (mean fold changes calculated with the RQ manager v.1.2 software) in patients: miR-181c, miR-331-5p, miR-193a-3p, miR-196b, miR454, miR-125a-3p, and miR-137. In a second step, the seven candidate miRNAs were individually assayed in 31 patients and 25 controls (including the individuals used to create the pools). Briefly, each pre-amplification was quantified by duplicate with custom Taqman assays in an ABI 7500 Real-Time PCR (Applied Biosystems) and normalized against miR-191. Each patient's miRNA was compared with the level of the miRNA in a pool of all the controls. Only miR-331-5p mean RQ value was significantly higher in the patients compared to the controls (1,905 vs. $88 ; p=0.001 ;$ Mann-Whitney's $U$ test) (Fig. 1).

Some miRNAs have been proposed as plasma biomarkers for neurodegenerative disorders and processes affecting the central nervous system [12, 13]. However, to our knowledge no studies describing the plasma miRNA profile in plasma from PD patients have been reported. Two studies with blood cells and total blood were recently published. The first one determined the expression profile in peripheral blood mononuclear cells (PBMCs) of 19 patients and 13 controls and identified several miRNAs deregulated in this cell type [14]. The other one identified several candidate miRNAs in total peripheral blood [15]. None of the candidate miRNAs identified in these studies were found to be overrepresented in our patients. These

Table 1 MiRNA-331-5p predicted target genes and likely related pathways

\begin{tabular}{|c|c|c|c|}
\hline Pathway & Gene & $-\ln (p$ value $)$ & Pathway ID \\
\hline Axon guidance & SRGAP3, EPHA4, GNAI1 & 4.3 & hsa04360 \\
\hline Insulin signaling pathway & PPARGC1A, SHC3, PPP1R3A & 3.8 & hsa04910 \\
\hline Chronic myeloid leukemia & SHC3, SMAD4 & 2.7 & hsa05220 \\
\hline TGF-beta signaling pathway & SMAD6, SMAD4 & 2.2 & hsa04350 \\
\hline Keratan sulfate biosynthesis & B3GNT1 & 2.1 & hsa00533 \\
\hline Alkaloid biosynthesis II & MYST4 & 1.7 & hsa00960 \\
\hline Glycosphingolipid biosynthesis-neo-lactoseries & B3GNT1 & 1.5 & hsa00602 \\
\hline MAPK signaling pathway & ATF2, MEF2C, DUSP1 & 1.5 & hsa04010 \\
\hline 1- and 2-Methylnaphthalene degradation & MYST4 & 1.4 & hsa00624 \\
\hline Tight junction & CASK, GNAI1 & 1.2 & hsa04530 \\
\hline Phenylalanine metabolism & MYST4 & 1.2 & hsa00360 \\
\hline Benzoate degradation via CoA ligation & MYST4 & 1.1 & hsa00632 \\
\hline Limonene and pinene degradation & MYST4 & 1.1 & hsa00903 \\
\hline Aminosugars metabolism & GNPDA2 & 1.1 & hsa00530 \\
\hline
\end{tabular}


discrepancies could be mainly explained by the fact that the three studies analyzed different blood sample types: purified cells, total blood and plasma. In addition, these studies would exclude blood-cell origin for this PD-circulating miRNA.

Finally, we performed a bioinformatic analysis of genes that are predicted to have miR-331-5p target sites in their $3^{\prime}$ UTR and identified several candidates that were implicated in molecular pathways associated with the nervous system (DIANA miRPath 2.0; http://diana.cslab.ece.ntua.gr/) (Table 1). Among others, the pathways related with neurogenesis and neurodegeneration could be involved.

In conclusion, we found miR-331-5p as a possible circulating miRNA in PD patients. This miRNA could be useful for diagnostic purposes or monitoring disease progression and treatment responses. However, our study was based on a limited number of patients at onset stage and requires further replication in larger cohorts of patients at different disease stages.

Acknowledgments Authors thank the "Fundacion Parkinson Asturias" and "Obra Social Cajastur" for their support. This work was supported by grant 11/0093 from the Spanish "Fondo de Investigaciones Sanitarias-Fondos FEDER" European Union. LFC and LDM are predoctoral fellowships of FICYT-Principado de Asturias.

Conflicts of interest The authors declare no conflicts of interest.

\section{References}

1. Braak H, Ghebremedhin E, Rub U, Bratzke H, Del Tredici K (2004) Stages in the development of Parkinson's disease-related pathology. Cell Tissue Res 318:121-134

2. Nuytemans K, Theuns J, Cruts M, Van Broeckhoven C (2010) Genetic etiology of Parkinson disease associated with mutations in the SNCA, PARK2, PINK1, PARK7, and LRRK2 genes: a mutation update. Hum Mutat 31:763-780

3. Eacker SM, Dawson TM, Dawson VL (2009) Understanding microRNAs in neurodegeneration. Nat Rev Neurosci 10:837-841
4. Kim J, Inoue K, Ishii J, Vanti WB, Voronov SV, Murchison E, Hannon G, Abeliovich A (2007) A MicroRNA feedback circuit in midbrain dopamine neurons. Science 317:1220-1224

5. Wang G, van der Walt JM, Mayhew G, Li YJ, Zuchner S, Scott WK, Martin ER, Vance JM (2008) Variation in the miRNA-433 binding site of FGF20 confers risk for Parkinson disease by overexpression of alpha-synuclein. Am J Hum Genet 82:283-289

6. Junn E, Lee KW, Jeong BS, Chan TW, Im JY, Mouradian MM (2009) Repression of alpha-synuclein expression and toxicity by microRNA-7. Proc Natl Acad Sci USA 106:13052-13057

7. Gehrke S, Imai Y, Sokol N, Lu B (2010) Pathogenic LRRK2 negatively regulates microRNA-mediated translational repression. Nature 466:637-641

8. Minones-Moyano E, Porta S, Escaramis G, Rabionet R, Iraola S, Kagerbauer B, Espinosa-Parrilla Y, Ferrer I, Estivill X, Marti E (2011) MicroRNA profiling of Parkinson's disease brains identifies early downregulation of miR-34b/c which modulate mitochondrial function. Hum Mol Genet 20:3067-3078

9. Reid G, Kirschner MB, van Zandwijk N (2011) Circulating microRNAs: association with disease and potential use as biomarkers. Crit Rev Oncol Hematol 80:193-208

10. Olivieri F, Spazzafumo L, Santini G, Lazzarini R, Albertini MC, Rippo MR, Galeazzi R, Abbatecola AM, Marcheselli F, Monti D, Ostan R, Cevenini E, Antonicelli R, Franceschi C, Procopio AD (2012) Age-related differences in the expression of circulating microRNAs: miR-21 as a new circulating marker of inflammaging. Mech Ageing Dev 133:675-685

11. Hughes AJ, Daniel SE, Kilford L, Lees AJ (1992) Accuracy of clinical diagnosis of idiopathic Parkinson's disease: a clinicopathological study of 100 cases. J Neurol Neurosurg Psychiatry 55:181-184

12. Geekiyanage H, Jicha GA, Nelson PT, Chan C (2012) Blood serum miRNA: non-invasive biomarkers for Alzheimer's disease. Exp Neurol 235:491-496

13. Li X, Khanna A, Li N, Wang E (2011) Circulatory miR34a as an RNA based, noninvasive biomarker for brain aging. Aging (Albany NY) 3:985-1002

14. Martins M, Rosa A, Guedes LC, Fonseca BV, Gotovac K, Violante S, Mestre T, Coelho M, Rosa MM, Martin ER, Vance JM, Outeiro TF, Wang L, Borovecki F, Ferreira JJ, Oliveira SA (2011) Convergence of miRNA expression profiling, alpha-synuclein interaction and GWAS in Parkinson's disease. PLoS ONE 6:e25443

15. Margis R, Rieder CR (2011) Identification of blood microRNAs associated to Parkinson's disease. J Biotechnol 152:96-101 